\title{
A spectroscopic and photometric study of the planetary nebulae Kn 61 and Pa 5
}

\author{
Ma. T. García-Díaz ${ }^{1}$, D. González-Buitrago ${ }^{1}$, J. A. López ${ }^{1}$, S. Zharikov ${ }^{1}$, G. Tovmassian ${ }^{1}$, \\ N. Borisov' ${ }^{2}$, G. Valyavin ${ }^{2}$ \\ tere, dgonzalez, jal, zhar, gag@astro.unam.mx, borisov@sao.ru, \\ gvalyavin@gmail.com
}

Received __; accepted _

\footnotetext{
${ }^{1}$ Instituto de Astronomía, Universidad Nacional Autónoma de México. Km 103 Carretera Tijuana-Ensenada, 22860 Ensenada, Baja California, México

${ }^{2}$ Special Astrophysical Observatory of the RAS, 369167, Nizhny Arkhyz, KarachaevoCherkesia, Russia
} 


\begin{abstract}
We present the first morpho-kinematical analysis of the planetary nebulae Kn 61 and $\mathrm{Pa} 5$ and explore the nature of their central stars. Our analysis is based on high resolution and medium resolution spectroscopic observations, deep narrow-band imaging, and integral photometry. This material allows us to identify the morphological components and study their kinematics. The direct images and spectra indicate an absence of the characteristic [N $\mathrm{II}]$ and $[\mathrm{S}$ II] emission lines in both nebulae. The nebular spectrum of Kn 61 suggests an hydrogen deficient planetary nebula and the stellar spectrum of the central star reveals a hydrogen deficient PG 1159-type star. The [O III] position velocity diagram reveals that Kn 61 is a closed, empty, spherical shell with a thin border and a filamentary surface expanding at $67.6 \mathrm{~km} \mathrm{~s}^{-1}$ and the shell is currently not expanding isotropically. We derived a kinematic age of $\sim 1.6 \times 10^{4}$ yrs for an assumed distance of $4 \mathrm{kpc}$. A photometric period of $\sim 5.7( \pm 0.4)$ days has been detected for $\mathrm{Kn}$ 61, indicating presence of a possible binary system at its core. A possible link between filamentary, spherical shells and PG 1159-type stars is noted.

The morphology of $\mathrm{Pa} 5$ is dominated by an equatorial toroid and faint polar extensions. The equatorial region of this planetary nebula is expanding at $45.2 \mathrm{~km} \mathrm{~s}^{-1}$. The stellar spectrum corresponds to a very hot star and is dominated by a steep blue rising continuum and He II, Balmer and Ca II photospheric lines.
\end{abstract}

Subject headings: Planetary Nebulae: individual (Kn 61, Pa 5) - ISM: kinematics techniques: spectroscopy, photometry, imagery 


\section{Introduction}

In the present study we analyze the characteristics of two planetary nebulae, namely, Kn 61 which has a nearly perfect spherical shape and $\mathrm{Pa} 5$, which presents a toroidal structure with faint polar extensions. We also explore the possibility that these PNe host binary cores, as has been suggested by Long et al. (2013), Kronberger et al. (2011), Douchin et al. (2011) and Jacoby et al. (2010).

The PN Kn 61 (nicknamed the Soccer Ball Nebula) was discovered by Kronberger et al. (2011). A high quality optical image in the combined light of [O III] $5007 \AA$ and H $\alpha$ has been published by the GEMINI observatory (http://www.gemini.edu/node/11656). Of particular interest is that this object lies within the Kepler field. Long et al. (2013) report brightness variability with a possible periodicity up to $\sim 6$ days from Kepler data. The composite [O III] and H $\alpha$ Gemini image (see Figure 1 from Douchin et al. 2011) reveals a predominantly bright [O III] filamentary, spherical, shell. The central star of Kn 61, also known as SDSS J192138.93+381857.2 has magnitude $u=18.66( \pm 0.01), g=$ 18.03( \pm 0.01$), r=18.48( \pm 0.01)$. The (uncertain) distance has been assumed to be $4 \mathrm{kpc}$ (http://www.gemini.edu/node/11656), from the GEMINI note on the object, mentioned above, though given its angular size of nearly 110 arcsec it is possible that it could be slightly closer.

Pa 5 was discovered by D. Patchick (Jacoby et al. 2010). This nebula also lies within the Kepler field. Østensen, et al. (2010) using low resolution spectroscopy in the spectral range $3500-5300 \AA$, found that the central star of Pa $5, \mathrm{~J} 19195+4445$, is a very hot one. They identify C IV and He II lines in the 4640 - $4686 \AA$ region in the stellar spectrum as indicative of the PG 1159 class. In addition, they find a period of 1.12 days using the Kepler data. The origin of variability of the central star $(\approx 0.002 \mathrm{mag})$ is reported as unknown. Kronberger et al. (2006) have classified Pa 5 as a probable bipolar PN. In direct images 
(DSS) it looks like a bipolar but only the northeast lobe is apparent with the opposite lobe being much fainter.

In order to improve our understanding of these two PNe contained within the Kepler field we present new spectroscopic and CCD imaging and photometric results for the nebulae and their central stars. This work combines results from datasets obtained at the Observatorio Astronómico Nacional at San Pedro Mártir, B. C., México (OAN-SPM) and from the Special Astrophysical Observatory 6-m telescope (SAO-RAS).

The structure of the paper is as follows. In $₫ 2$ we describe the observations and the data-reduction steps. In $₫ 3$ we discuss the results. In 94 we summarize the conclusion of this work.

\section{The Observations}

Tables 1 and 2 display the information relative to the observing runs, divided into instruments with respective dates, exposure times, number of spectra and spectral range. All data were reduced using standard procedures in IRAF1 to correct bias, cosmic rays extract and wavelength calibrate the one and two-dimensional spectra.

\subsection{Optical Imaging and Photometry}

The monochromatic images of Kn 61 and Pa 5 were obtained on 2012, August 3 for Kn 61, and on 2013, August 9 for Pa 5, using the Mexman CCD photometer on the $84 \mathrm{~cm}$

\footnotetext{
${ }^{1}$ IRAF is distributed by the National Optical Astronomy Observatories, which is operated by the Association of Universities for Research in Astronomy, Inc. under cooperative agreement with the National Science foundation
} 
telescope at the OAN-SPM. We used the ESOPO CCD detector with $2048 \times 4612$ square pixels, $15 \mu \mathrm{m}(0.25 \times 0.25$ arcsec $)$ pixel size. Kn 61 and $\mathrm{Pa} 5$ were observed in the light of

[O III] $5007 \AA, \mathrm{H} \alpha,[\mathrm{S} \mathrm{II}] 6731 \AA$ and [N II] $6584 \AA$ with bandwidths of $52 \AA, 11 \AA, 54 \AA$ and $10 \AA$, respectively. The exposure time for each filter was $1800 \mathrm{~s}$. The data were corrected for bias, cosmic rays hits and flat-fielded in order to remove the pixel-to-pixel response.

Figures 1 and 2 are mosaics of images of Kn 61 and Pa 5, respectively. For each figure, the panels correspond to [O III] (left panel), and $\mathrm{H} \alpha$ (right panel). We did not show the $[\mathrm{N}$ II] nor [S II] images because the nebulae do not have any emission in these lines.

Kn 61 and Pa 5 were also observed with the $1.5 \mathrm{~m}$ telescope at the OAN-SPM, and the RATIR camera (Butler et al. 2012), in the $r$ and $i$ filters simultaneously, for 18 consecutive days with half hour observations per night per object. The exposure time was 90 seconds for each filter.

We performed differential photometry using the IRAF package apphot selecting comparison stars in the field for Kn 61 and $\mathrm{Pa} 5$.

\subsection{Spectroscopy Observations}

\subsubsection{High resolution}

Long-slit high-resolution spectroscopic observations of the nebulae Kn 61 and $\mathrm{Pa} 5$ were obtained at the OAN-SPM, México, on 2012, May 25, and are available in "The San Pedro Mártir Kinematic Catalogue of Galactic Planetary Nebulae" (López et al. 2012).

The data were obtained using the Manchester Echelle Spectrometer, MES-SPM, (Meaburn et al. 2003) on the $2.1 \mathrm{~m}$ telescope in its $f / 7.5$ configuration The MES-SPM is a long-slit, echelle spectrometer that has no cross-disperser; it isolates single orders 
using interference filters. For the present observations we used two filters of $90 \AA$ and $50 \AA$ bandwidths to isolate the 87th and 114th orders containing the $\mathrm{H} \alpha+[\mathrm{N}$ II $] 6584 \AA$, and [O III] $5007 \AA$ nebular emision lines, respectively. This instrument was equipped with a Marconi CCD detector with $2048 \times 2048$ square pixels, each $13.6 \mu \mathrm{m}$ on a side. The detector was set to a binning of $3 \times 3$ in both the spatial and spectral directions.

We used a $150 \mu \mathrm{m}$ slit of 1".9 and 5!47 projected width and length, respectively. The resultant spectral dispersion was approximately $0.26 \AA$ pixel $^{-1}$ and $0.19 \AA$ pixel $^{-1}$ at $\mathrm{H} \alpha$ and $[\mathrm{O}$ III $] 5007 \AA$, respectively (equivalent to $\sim 11.5 \mathrm{~km} \mathrm{~s}^{-1}$ ). For Kn 61 we placed the slit at a position angle, P.A. $=0^{\circ}$ and through the central star of the nebula (see Figure 1). For $\mathrm{Pa} 5$ we placed the slit along the bright elongated structure whose axis is oriented at P.A. $=50^{\circ}$ (see Figure 2). A second exposure along the axis defined by the lobes was obtained but it turned out of poor signal to noise due to thick clouds. Images of the slit projected on the sky were obtained to establish the exact position of the slit on the target. Wavelength calibration against the spectrum of a Th/Ar lamp was obtained after each science exposure, this yields an accuracy of $\pm 1 \mathrm{~km} \mathrm{~s}^{-1}$ when converted to radial velocity. Spectra presented in this work are corrected to heliocentric velocity $\left(V_{\odot}\right)$. The bi-dimensional $\mathrm{H} \alpha$ and $[\mathrm{O}$ III] $5007 \AA$ emission line spectra or position-velocity (P-V) arrays are shown in Figures 3 and 4 for $\mathrm{Kn} 61$ and $\mathrm{Pa}$ 5, respectively.

\subsubsection{Medium resolution}

\section{$O A N-S P M$}

Medium resolution spectroscopy was performed with the $2.1 \mathrm{~m}$ telescope at the OAN-SPM in 2012 and 2013. We used the Boller \& Chivens (B\&Ch) spectrograph using a $600 \mathrm{l} \mathrm{mm}^{-1}$ grating with a dispersion of $1.17 \AA$ pixel $^{-1}$. The spectra span the wavelength range from 
$4000 \AA$ to $7000 \AA$. We also used a $1200 \mathrm{l} \mathrm{mm}^{-1}$ grating with a dispersion $0.60 \AA$ pixel $^{-1}$ covering the wavelength range from $4560 \AA$ to $5750 \AA$. A Marconi CCD with $2048 \times$ 2048 pixels and no binning was used. In all observations the slit was oriented east-west and on the central star for each planetary nebula. The exposure time was $1200 \mathrm{~s}$ per spectrum. A He-Ne-Ar comparison lamp was obtained after every third spectra to ensure good wavelength calibration. For flux calibration a standard star was observed at the end of the night, taken from the list of Landolt (1992) and Bohlin et al. (2001).

Figures 5 y 6 are mosaics of the stellar and nebular spectra for Kn 61 and $\mathrm{Pa} 5$ respectively, taken from OAN-SPM. The lefthand panels show medium resolution spectra of the central star, while the righthand panels show the nebular spectra. Panels a) and b) were taken with the $600 \mathrm{l} \mathrm{mm}^{-1}$ grating, while the lower panels, panels c) and d), were taken with the $12001 \mathrm{~mm}^{-1}$ grating. The $1200 \mathrm{l} \mathrm{mm}^{-1}$ grating spectra shown in these figures were averaged over a single night of observation to improve the signal-to-noise. The spectra for the nebulae were extracted from the full length of the slit that covers the nebulae.

\section{$S A O-R A S$}

Medium-resolution spectroscopic data of Kn 61 were recently obtained at the Special Astrophysical Observatory 6-m telescope on 2013, September 12, using the SCORPIO spectrograph (Afanasiev \& Moiseev 2005) installed at the prime focus of the SAO 6-m telescope of the Russian Academy of Sciences in the long-slit unit mode. In this mode, the slit dimensions are $6^{\prime}$ long $\times 1^{\prime \prime}$ wide. We used the CCD detector EEV42-40 and the grism VPHG $1200 \mathrm{~g}$ (1200 lines $\mathrm{mm}^{-1}$ with a spectral dispersion of $\left.0.88 \AA \mathrm{pixel}^{-1}\right)$.

Three spectra were taken in the spectral range between $3900 \AA$ and $5700 \AA$. The standard star (BD28d4211) was observed for flux calibration. The calibrated spectra are shown in Figure 7. 


\section{Results and Discussion}

\subsection{Kn 61}

Figure 1 shows the images of $\mathrm{Kn} 61$ in [O III], and $\mathrm{H} \alpha$. We did not detect any emission in $[\mathrm{N}$ II] $6584 \AA$ nor in $[\mathrm{S} \mathrm{II}] 6717,6731 \AA$ in our images. The [O III] image shows a highly filamentary bubble. The $\mathrm{H} \alpha$ image is extremely faint showing some limb brightening at the border. The outline of the bubble in the [O III] and $\mathrm{H} \alpha$ images cover the same area. In other hydrogen-deficient nebulae, such as Abell 30 (e.g. Meaburn \& López 1996) and Abell 78 (e.g. Meaburn et al. 1998), the faint hydrogen shell clearly extends beyond the [O III] shell indicating that the latter was expelled in a late thermal flash once the hydrogen layer of the central star had been consumed and the hydrogen-rich envelope had already expanded. In the case of Kn 61 the nebular emission points towards an already hydrogen impoverished shell as it left the AGB.

The echelle, long-slit, spectra of the nebular shell of Kn 61 (see Figure 3) confirm the same peculiar line emission ratios in the sense that the $\mathrm{H} \alpha$ emission line is extremely faint and the $[\mathrm{N}$ II] lines are absent whereas [O III] is bright. The line profiles (see Figure 3) indicate that Kn 61 is a closed, hollow, spherical shell with a thin border. The filamentary surface is revealed in the [O III] line profile by the knotty structure along the thin border of the profile. From the [O III $] \mathrm{P}-\mathrm{V}$ array we find that the velocity splitting at the center of the profile amounts to $135.3 \mathrm{~km} \mathrm{~s}^{-1}$ with the blueshift component at $V_{\odot}=-93.0 \mathrm{~km} \mathrm{~s}^{-1}$ and the redshift component at $V_{\odot}=+42.3 \mathrm{~km} \mathrm{~s}^{-1}$. This yields an expansion velocity of $67.6 \mathrm{~km} \mathrm{~s}^{-1}$, which is high for PNe like this (Pereyra et al. 2013). The systemic heliocentric velocity, as measured from the midpoint between the line splitting is $-25.4( \pm 2) \mathrm{km} \mathrm{s}^{-1}$. A departure from perfect spherical or isotropic expansion is indicated by a small but definitive tilt in the line profiles. This tilt runs from $-29.73 \mathrm{~km} \mathrm{~s}^{-1}$ in the south (bottom of the profile) to $-12.07 \mathrm{~km} \mathrm{~s}^{-1}$ (top) in the north. This must be produced by a slightly 
anisotropic expansion of the bubble, i.e. receding velocities in the northern part dominate over the approaching ones in the southern section of the bubble, with respect to the systemic velocity. If this trend continues it is then expected that Kn 61 will become an elongated nebula, tending to an elliptical shape with time. A crude estimate of the kinematic age for the nebula is calculated considering that the angular diameter of the nebula is $104^{\prime \prime}$. For an assumed distance of $4 \mathrm{kpc}$, its linear radius is $0.96 \mathrm{pc}$. Considering as constant the expansion velocity quoted above, this yields a kinematic age for the bubble of $1.6 \times 10^{4} \mathrm{yrs}$.

Likewise, the medium resolution nebular spectrum for Kn 61 (Figure 5, righthand panels) shows only [O III] 4959,5007 $\AA, \mathrm{H} \alpha$ and very weak HeII $4686 \AA$ and $\mathrm{H} \beta$ nebular emission lines. The sky lines have been removed. The nebular medium-resolution spectrum from the SAO-RAS (Figure 7, right panel) confirms the previous results for the corresponding wavelength ranges. A list of the main emission lines and their fluxes are presented in Table 3. The nebular spectrum of Kn 61 does not show measurable [O III $] 4363 \AA$ neither the $[\mathrm{N} \mathrm{II}]$ lines. No other diagnostic line ratios are available within this wavelength range, therefore it is not possible to derive electron temperature nor electron density and consequently ionic abundances. It is interesting to notice however that the low $\mathrm{H} \alpha / \mathrm{H} \beta$ flux ratio measured seems to indicate that $\mathrm{Kn} 61$ is at least partially optically thin to Lyman photons (e.g. Osterbrock, 1974) which would contribute to explain the very faint $\mathrm{H} \alpha$ emissivity of this nebula.

\subsubsection{Central star of Kn 61}

Figure 5 (lefthand panels) shows the spectra from OAN-SPM of the central star of Kn 61 . The spectrum has been binned $\times 4$ in the spectral axis to improve signal to noise and help highlight photospheric lines. The stellar spectra show lines characteristic of a Group $3=$ lg E (low gravity central star) class of PG 1159 star (e.g. Parthasarathy, Acker \& Stenholm 
1998), revealed by the absorption line at C IV $4647 \AA$ and the double emission lines also from C IV 5801,5812 A. These lines are more clearly appreciated in the medium resolution stellar spectrum of Kn 61 obtained at SAO-RAS (see Figure 7, left panel). The later spectrum for the central star indicates the presence of an emission line at $4713 \AA$ this is likely a nebular contamination from either He I or [Ar IV], as it is sometimes observed in other PG 1159 stars (Parthasarathy, Acker \& Stenholm 1998). Additionally the C IV $4647 \AA$ absorption line is detected. The PG 1159 stars are considered to be very hot, hydrogen deficient post-AGB stars. In a recent survey of such stars detected in the Data Release 10 of the Sloan Digital Sky Survey (Werner et al. 2014) seven stars are of PG1159-type with spectra similar to the central star of Kn 61. Particular close resemblance is found between the spectra of the central star of Kn 61 and SDSS J075415.12+085232.18, a PG1159 star with $120000 \mathrm{~K}, \log g=0.33$ and $\mathrm{C} / \mathrm{He}=0.33$, where the $\mathrm{C}$ IV $4647 \AA$ has also been identified.

We have monitored the photometric behavior of the central star of Kn 61 for 2-3 hours each night for 16 consecutive nights. No strong amplitude or periodic variability beyond the natural data scatter on the order of 0.028 mag was detected on such short time scales. Therefore, the photometric data were averaged for each night and the resulting light curve covering 16 nights was analyzed for the presence of a periodic signal using the Period04 program. The latter employs a discrete Fourier transformation technique to calculate the power spectra and spectral window corresponding to the analyzed time-series. This produced a statistically significant peak at a frequency of 0.176 days $^{-1}$ in the power spectrum (see Figure 8 left panel), which corresponds to a period of $5.7( \pm 0.4)$ days. The semi-amplitude of variability is $\mathrm{A} \approx 0.02 \mathrm{mag}$ and is comparable with the root mean square (RMS) of individual nightly light curves before averaging. Similar analysis on two field stars of comparable brightness proved that the periodic signal is authentic and far exceeds random noise. The significance level corresponding to $99 \%$ is marked in Figure 8 by a 
horizontal dashed line. In addition we frequency folded the power spectrum within the spectral window, which helps to sort out the alias periods produced by uneven time series. The result confirms a strong, single peak at the indicated frequency. The light curve folded with the determined period is presented in the right panel of Figure 8. The best fit sin curve with the $\mathrm{A}=0.0176$ amplitude around the average magnitude $\mathrm{m}_{\mathrm{R}}=18.674$ is also plotted. The phase zero is set arbitrarily to the moment when the object has an average brightness. The error bars in Figure 8 (right panel) reflect the RMS of individual points around each night's average and they far exceed statistical errors of aperture photometry.

Long et al. (2013) analyzed the light curve of the central star of Kn 61 and found cyclical variability which might be periodic on time scales from 2 to 6 days. Our result does not contradict their non-conclusive findings. The object requires longer monitoring and more uniform observations to determine the origin of the variability.

It is interesting to notice, however, that for more than a couple of decades binary nuclei in PNe have been suggested as responsible for shaping non-spherical, axis-symmetric or point-symmetric nebulae (e.g. Livio \& Soker 1988) and as a source of their collimated outflows. Kn 61 does not display collimated outflows and it is close to spherical symmetry. However, if the central star of Kn 61 is a binary, its possible orbital period of $\approx 6$ days is relatively long compared to other binary CSPN. The majority of close binary CSPN have usually periods of only a fraction of a day (De Marco 2009). A longer period means that the secondary is relatively distant, leading to reduced chance of interaction between stars and thus reduced influence on the nebular shape and collimation of an outflow.

It is also of interest to compare Kn 61 with the PNe NGC 7094 and Abell 43 (Rauch et al. 2005). These PNe have a very similar morphological structure to Kn 61, i.e. they are both roughly spherical filamentary bubbles and in both cases their central stars are classified as hybrid-PG 1159 type (Solheim et al. 2007). They also display high expansion 
velocities, $\geq 50 \mathrm{~km} \mathrm{~s}^{-1}$. These stars are thought to have experienced a late thermal pulse while they were still on the asymptotic giant branch and hydrogen-shell burning was still on, yet they are able to keep small amounts of atmospheric hydrogen. We have drawn the images and spectra of these PNe from the "The San Pedro Mártir Kinematic Catalogue of Galactic Planetary Nebulae" (López et al. 2012) and these are reproduced in Figure 9. Notice the remarkable similarity with $\mathrm{Kn} 61$, both in the images and the bi-dimensional line profiles. In these cases the $\mathrm{H} \alpha$ line is bright, in contrast with Kn 61. Also in both cases the [N II] lines are absent, as in Kn 61 (see López et al. 2012). Pereyra et al. (2013) found from an analysis of 100 evolved PNe that those objects located at the point of maximum temperature in the evolutionary tracks, right before their luminosity starts dropping towards the white dwarf region, exhibit a lack of [N II] emission. The lack of $[\mathrm{N} \mathrm{II}]$ is a consequence of the small fraction of nitrogen in the singly ionized state. This, in turn, follows from the high ionization levels of the nebula due to a very hot central star that photoionizes most of the $\mathrm{N}$ into doubly and triply ionized states. The relation between soap bubbles shapes, i.e. highly filamentary spherical bubbles, and the PG 1159 type stars is puzzling and is pointed out here for the first time.

\subsection{Pa 5.}

Figure 2 shows the corresponding images for Pa 5 . We did not detect any emission in [N II] $6584 \AA$ nor in [S II] 6717,6731 $\AA$ in our images. The [O III] and $\mathrm{H} \alpha$ images show a bright bar that looks like an equatorial structure with a faint lobe extending perpendicular to it towards the northeast. A much fainter extension is barely appreciated on the opposite side. The slit was located along the bright structure. The $\mathrm{P}-\mathrm{V}$ arrays (see Figure 4) from this position confirm that this structure has an expanding doughtnut-like or toroidal shape, expanding at $45.2( \pm 2) \mathrm{km} \mathrm{s}^{-1}$ and with a systemic velocity of $12.4( \pm 2) \mathrm{km} \mathrm{s}^{-1}$. The $\mathrm{H} \alpha$ 
line profile looks filled close to the top and bottom cusps indicating that the toroid has a thick inner wall (see Figure 2, right panel). Unfortunately weather conditions precluded us from obtaining a second slit position along the polar extensions that would have help characterized the likely bipolar structure, as suggested by Kronberger et al. (2006). In a study of PNe with close binary nuclei López et al. (2011) find that the large majority of these objects tend to have equatorial density enhancements likely produced by the ejection of the envelope along the equatorial plane after the common envelope phase. If the nucleus of $\mathrm{Pa} 5$ is confirmed as a close binary system (see below) its morphology would agree with this characteristic.

The one-dimensional medium-resolution nebular spectra of Pa 5 are shown in Figure 6 (panels b \& d). The emission lines H $\alpha$, [O III] 5007,4959 $\AA, \mathrm{H} \alpha, \mathrm{H} \beta, \mathrm{H} \gamma$, He II 4686,5412 $\AA$ and $[$ Ar IV] 4740,4711 $\AA$ stand out in the spectra. A list of the main emission lines and their fluxes are presented in Table 3. As for Kn 61 the [O III] $4363 \AA$ line is too weak to measure and the [N II] lines are absent, it is therefore not possible to derive a relieble electron temperature in the available wavelength range. For the case of the electron density, the $[\mathrm{Ar} I V]$ lines are present and their line ratio indicates an electron density of the order of $1100( \pm 200) \mathrm{cm}^{-3}$, assuming an electron temperature of $10^{4} \mathrm{~K}$. This relative high density is reasonably consistent with the fact that the spectrum comes from the dense waist or equator of this $\mathrm{PN}$.

\subsubsection{Central star of $\mathrm{Pa} 5$}

Østensen, et al. (2010) using low resolution spectroscopy in the spectral range of $3500-5300 \AA$, found that the central star of Pa 5 , J19195+4445, is a very hot star and they identify it as belonging to the PG 1159 on the basis of the presence of C IV and He II

close to $4686 \AA$. The spectra for the central star of Pa 5, shown in Figure 6 (panel a \& c) 
reveal the presence of lines from the Pickering series He II 4200, 4541, 4686, $5412 \AA ; \mathrm{H} \beta$, $\mathrm{H} \gamma$ and $\mathrm{Ca}$ II $5889 \AA$, but we do not detect the C IV lines so we are unable to confirm the PG 1159 nature of this star.

In addition, Østensen et al. (2010) using the Kepler data find a period of 1.12 days for the central star of $\mathrm{Pa} 5$. They report a very small amplitude of only $0.05 \%$ in terms of the significance of the variability and consider the origin of the variability as unknown. Although we attempted to measure radial velocity and photometric variations over several nights, the period of this object being close to one day precludes observing different phases from a single ground-based telescope and we could not detect any orbital modulation.

\section{Conclusions}

Kn 61 is a higly filamentary bubble with a high expansion velocity, $67.6 \mathrm{~km} \mathrm{~s}^{-1}$. Kn 61 does not show emission in [N II], and [S II] due to a very hot central star that photoionizes most of the $\mathrm{N}$ into doubly and triply ionized states. The $\mathrm{H} \alpha$ emission is extremely faint in comparison to [O III], showing some limb brightening at the border of the nebula. The nebular and stellar emission of Kn 61 suggests that the central star had nearly exhausted its hydrogen layers as it left the AGB. From medium-resolution spectra of the central star, we detected the absorption line at C IV $4647 \AA$, the emission line C IV $4659 \AA$, and the double emission lines also from C IV 5801,5812 A, which are characteristic of PG 1159 hydrogen deficient, post-AGB stars. The morphology of Kn 61 is very similar to NGC 7094 and Abell 43, whose nebular spectrum has the same kinematic behavior as that of Kn 61 . None of these nebulae present [N II] emission lines, though the $\mathrm{H} \alpha$ line emission is bright in NGC 7094 and Abell 43, in contrast to Kn 61. NGC 7094 and Abell 43 have central

stars of hybrid-PG 1159 type, they both are pulsators (Solheim et al. 2007), they present He II/C IV 4650-4686 $\AA$ and and more prominent Balmer lines in the associated nebula. 
These objects, including Kn 61, are likely mature nebulae located in the region of maximum temperature in their corresponding evolutionary tracks in the Hertzsprung Russell diagram, right before their luminosity starts dropping towards the white dwarf region. The lack of $[\mathrm{N}$ II] emission is a characteristic of PNe in this stage of evolution, since low ionization states are pumped to higher levels and is also where the largest expansion velocities are observed, as pointed out by Pereyra et al. (2013) . The possible link between filamentary bubbles and PG 1159-type stars is noted for the first time and requires additional analysis. From the CCD photometry performed on the central star of Kn 61 we obtained a light curve with an orbital period for the system of $\sim 5.7( \pm 0.4)$ days, this coincides with preliminary Kepler data presented by Long et al. (2013) and indicates the possible presence of a binary core, though longer monitoring and uniform observations are required to exclude other explanations, such as variations driven by pulsations. Although Kn 61 is close to having a spherical outline or shape, a deviation from spherical symmetry and isotropic expansion has been detected in the long-slit spectra. A crude kinematic age for the bubble yields $1.6 \times 10^{4}$ yrs.

Pa 5 does not show [N II], and [S II] emission lines either. Its morphology is dominated by a dense equatorial toroid and faint polar extensions. The [O III] and $\mathrm{H} \alpha \mathrm{P}-\mathrm{V}$ arrays show that equatorial enhancement is thick, sitting at a systemic velocity of $12.4( \pm 2) \mathrm{km} \mathrm{s}^{-1}$ and expanding at $45.2( \pm 2) \mathrm{km} \mathrm{s}^{-1}$. The central star is very hot, showing prominent He II 4541,4686,5412 $\AA, \mathrm{H} \beta$ and Ca II $5889 \AA$ absorption lines. Kepler data have yielded a period of 1.12 days for the central star with a very small amplitude (Østensen et al. 2010) the origin of this variability remains unknown. Our CCD photometry and spectroscopy could not confirm any orbital modulation in this case.

Support for this study was provided by CONACyT, México through a research studentship to D.G.B. This research has benefited from the financial support of PAPIIT- 
UNAM through project IB100613-RR160613. This research is based upon observations acquired at the Observatorio Astronómico Nacional in the Sierra San Pedro Mártir (OAN-SPM), Baja California, México and from the Special Astrophysical Observatory 6-m telescope of the Russian Academy of Sciences. We are grateful to the excellent support of the technical personnel at the OAN-SPM. We thank the anonymous referee for the constructive comments that improved the presentation of this work. 


\section{REFERENCES}

Afanasiev V. L., \& Moiseev A. V., 2005, Astron. Lett, 31, 194

Bohlin, R. C., Dickinson, M. E., \& Calzetti, D. 2001, ApJ, 122, 2118

Butler, N., Klein, C., Fox, O., et al. 2012, SPIE, 8446, 10B

De Marco, O. 2009, PASP, 121, 316

Douchin, D., Jacoby, G. H., De Marco, O., Howell, S. B. \& Kronberger, M. 2011, IAU, 283, 119

García-Segura, G., Langer, N., Rózyczka, M., \& Franco, J. 1999, ApJ, 517, 767

García-Díaz Ma. T., López, J. A., García-Segura, G., Richer, M. \& Steffen, W. 2008, ApJ, 676,402

Iben, I., Jr., Kaler, J. B., Truran, J. W., \& Renzini, A. 1983, ApJ, 264, 605

Jacoby, G. H. Kronberger, Matthias; Patchick, Dana; Teutsch, Philipp; Saloranta, Jaakko; Howell, Michael; Crisp, Richard; Riddle, Dave; Acker, Agns; Frew, David J.; Parker, Quentin A. 2010, PASA, 27, 156

Kronberger, M., George H. Jacoby, Robin Ciardullo, Richard D. Crisp, Orsola De Marco, Dimitri Douchin, David J. Frew, Dianne Harmer, Mike Howell, Steve B. Howell, Quentin A. Parker, Dana Patchick, Travis Rector, Dave Riddle and Philipp Teutsch. 2011, IAU, 283, 414

Kronberger M., Teutsch P., Alessi B. Steine, M., Ferrero, L., Graczewski, K., Juchert, M., Patchick, D., Riddle, D., Saloranta, J. 2006, A\&A, 447, 921,

Landolt, A. U. 1992, AJ, 104, 340 
Livio, M., \& Soker, N. 1988, ApJ, 329, 764

Long, J., Jacoby, G., De Marco, O., et al. 2013, American Astronomical Society Meeting Abstracts, 221, 249.07

López, J. A., Richer, M. G., García-Díaz, M. T., Clark, D. M., Meaburn, J., Riesgo, H., Steffen, W., \& Lloyd, M. 2012, RMxAA, 48, 3

López, J. A., García Díaz, Ma. T., Richer, M. G., Lloyd, M., Meaburn, J. 2011, APN5.confE, 335L (2011arXiv1101.5653L)

Meaburn, J., López, J. A., Gutiérrez, L., Quiróz, F., Murillo, J. M. \& Valdéz, J. 2003, RMxAA, 39, 185M

Meaburn, J., López, J. A., Bryce, M., Redman, M. P. 1998, A\&A, 334, 670M

Meaburn, J., \& J., López, J. A. 1996, ApJ, 472, L45.

Meaburn \& J., López. 1996, ApJ, 472, L45.

Østensen, R. H., Silvotti, R., Charpinet, S., Oreiro, R., Handler, G., Green, E. M., Bloemen, S., Heber, U.; Gansicke, B. T., Marsh, T. R., and 18 coauthors. 2010, MNRAS, 409, $1470 \mathrm{O}$

Osterbrock, D. E. 1974, in "Astrophysics of Gaseous Nebulae" W.H. Freeman \& Company, p. 86

Parthasarathy, M., Acker, A. \& Stenholm, B. 1998, A\&A 329, L9

Pereyra, Margarita, Richer, Michael G., López, José Alberto. 2013, ApJ, 771, 114P

Rauch, T., Werner, K., Ercolano, B \& Köppen, J. 2005, AIP Conference Proceedings 804, 99 

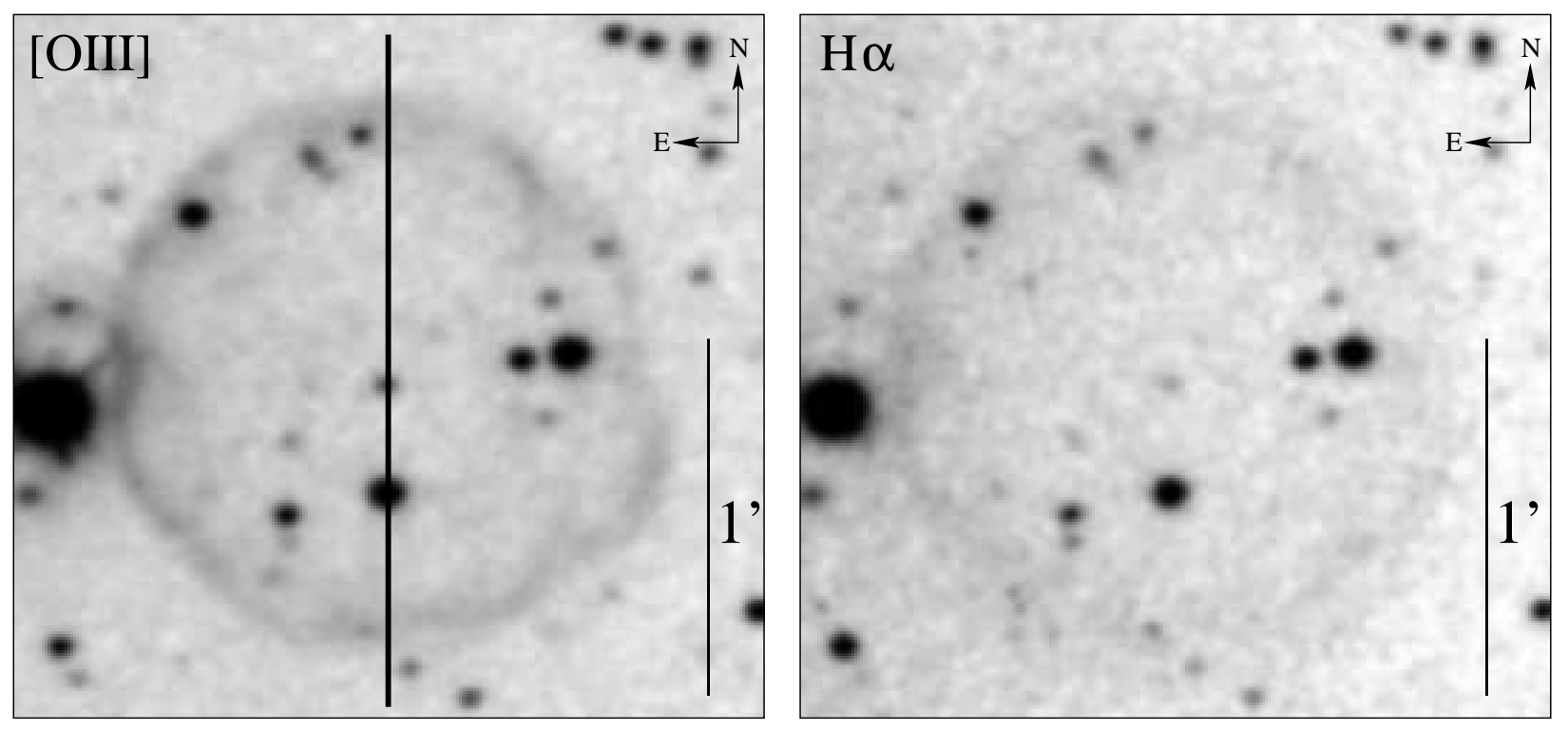

Fig. 1.- Images of Kn 61 obtained at the OAN-SPM $84 \mathrm{~cm}$ telescope, in the light of [O III] $5007 \AA$ (left panel) and $\mathrm{H} \alpha$ (right panel). Location of the long-slit position is indicated on the [O III] image.

Schwarz, U. J. 1978, A\&A, 65, 345

Solheim, J.-E.; Vauclair, G.; Mukadam, A. S.; Janulis, R.; Dobrovolskas, V. 2007, A\&A, $468,1057 \mathrm{~S}$

Werner, K., Rauch, T., \& Kepler, S. O. 2014, A\&A, 564, A53

Ziegler, M.; Rauch, T.; Reiff, E.; Werner, K.; Kruk, J. W.; Oliveira, C. 2008, ASPC, 391, $125 \mathrm{Z}$

This manuscript was prepared with the AAS IATEX macros v5.2. 
Table 1: Log of time-resolved observations of Kn61.

\begin{tabular}{l|cccc}
\hline & \multicolumn{4}{|c}{ Spectroscopy medium resolution/ } \\
\hline Date & No. of & Exp. time & Range & Resolution \\
DD/MM/20YY & frame & $(\mathrm{s})$ & $\AA$ & $\AA$ \\
\hline $11 / 08 / 13$ & 1 & 1200 & $4000-7000$ & 4.2 \\
$12 / 09 / 12$ & 2 & 1200 & $4560-5750$ & 2.0 \\
$17 / 07 / 12$ & 3 & 1200 & $4000-7000$ & 4.2 \\
$16 / 07 / 12$ & 1 & 1200 & $4560-5750$ & 2.0 \\
$09 / 06 / 12$ & 6 & 1200 & $4560-5750$ & 2.0 \\
\hline \hline
\end{tabular}

\begin{tabular}{|c|c|c|c|c|}
\hline & \multicolumn{4}{|c|}{ Spectroscopy medium resolution / 6.0m SAO-RAS } \\
\hline \multirow[t]{2}{*}{$12 / 09 / 13$} & 3 & 600 & $3900-5700$ & 5 \\
\hline & \multicolumn{4}{|c|}{ Spectroscopy hight resolution / 2.1m OAN-SPM } \\
\hline \multirow[t]{2}{*}{$25 / 05 / 12$} & 1 & 1800 & $\mathrm{H} \alpha,[\mathrm{O} \mathrm{III}]$ & $0.26(\mathrm{H} \alpha)$ \\
\hline & & & & $0.19([\mathrm{O}$ III $])$ \\
\hline
\end{tabular}

\begin{tabular}{l|ccl}
\hline \hline & \multicolumn{3}{|c}{ Photometry / 1.5m OAN-SPM } \\
\hline Date & No. of & Exp. time & filter \\
DD/MM/20YY & frame & $(\mathrm{s})$ & $\mathrm{R}$ \\
\hline $4,5,6,8,13 / 07 / 13$ & 19 & 90 & $\mathrm{R}$ \\
$14,16,17,31 / 07 / 13$ & 19 & 90 & $\mathrm{R}$ \\
$1,2,4,10 / 08 / 13$ & 20 & 90 & $\mathrm{R}$ \\
$11,13,28 / 08 / 13$ & 20 & 90 & H $\alpha,[\mathrm{O}$ III $]$ \\
\hline \hline
\end{tabular}


Table 2: Log of time-resolved observations of Pa5.

\begin{tabular}{l|cccc}
\hline & \multicolumn{4}{|c}{ Spectroscopy medium resolution/ } \\
\hline Date & No. of & Exp. time & Range & Resolution \\
DD/MM/20YY & frame & $(\mathrm{s})$ & $\AA$ & $\AA$ \\
\hline $16 / 07 / 12$ & 1 & 1200 & $4000-7000$ & 4.2 \\
$18,20,24 / 07 / 12$ & 17 & 1200 & $4250-5500$ & 2.0 \\
$12,14,15,16 / 09 / 12$ & 17 & 1200 & $4550-5750$ & 2.0 \\
$10,12 / 08 / 13$ & 3 & 1200 & $4000-7000$ & 4.2 \\
$11 / 08 / 13$ & 1 & 1200 & $4700-5700$ & 2.0 \\
$13,14,15 / 08 / 13$ & 22 & 1200 & $4450-5600$ & 2.0 \\
\hline \hline & Spectroscopy hight resolution / 2.1m OAN-SPM \\
\hline $25 / 05 / 12$ & 1 & 1800 & H $\alpha,[$ O III $]$ & $0.26(\mathrm{H} \alpha)$ \\
& & & & $0.19([\mathrm{O}$ III $])$ \\
\hline \hline
\end{tabular}

\begin{tabular}{l|ccl}
\hline \hline & \multicolumn{3}{|c}{ Photometry / 1.5m OAN-SPM } \\
\hline Date & No. of & Exp. time & filter \\
DD/MM/20YY & frame & $(\mathrm{s})$ & $\mathrm{R} / \mathrm{I}$ \\
\hline $5,6,8,13,14 / 07$ & $20 / 20$ & $90 / 90$ & $\mathrm{R} / \mathrm{I}$ \\
$16,17,28,30,31 / 07$ & $20 / 20$ & $90 / 90$ & $\mathrm{R} / \mathrm{I}$ \\
$1,2,4,10 / 08$ & $20 / 20$ & $90 / 90$ & $\mathrm{R} / \mathrm{I}$ \\
$11,13,14,28 / 08$ & $20 / 20$ & $90 / 90$ & $\mathrm{I}$ \\
$31 / 08$ & 20 & 90 & H $\alpha,[\mathrm{O}$ III] \\
\hline \hline
\end{tabular}


Table 3: Fluxes of the main emission lines

\begin{tabular}{|c|c|c|c|c|c|}
\hline \multicolumn{3}{|l|}{ Kn 61} & \multicolumn{3}{|l|}{ Pa 5} \\
\hline ID & $\begin{array}{c}\text { Wavelength Rest } \\
\AA\end{array}$ & $\begin{array}{l}\mathrm{F}_{\lambda} / \mathrm{F}(\mathrm{H} \beta) \\
\text { measured }\end{array}$ & ID & $\begin{array}{c}\text { Wavelength Rest } \\
\AA\end{array}$ & $\begin{array}{l}\mathrm{F}_{\lambda} / \mathrm{F}(\mathrm{H} \beta) \\
\text { measured }\end{array}$ \\
\hline He II & 4685.68 & 0.88 & $\mathrm{H} \mathrm{I}$ & 4340.47 & 0.53 \\
\hline O III & 4958.93 & 4.27 & He II & 4685.70 & 1.27 \\
\hline O III & 5006.85 & 14.42 & Ar IV & 4711.33 & 0.24 \\
\hline \multirow[t]{5}{*}{$\mathrm{H} \mathrm{I}$} & 6562.82 & 2.69 & Ar IV & 4740.19 & 0.18 \\
\hline & & & O III & 4958.93 & 1.35 \\
\hline & & & O III & 5006.85 & 4.16 \\
\hline & & & He II & 5411.57 & 0.14 \\
\hline & & & $\mathrm{H} \mathrm{I}$ & 6562.82 & 2.94 \\
\hline $\log \mathrm{F}(\mathrm{H} \beta)$ & \multicolumn{2}{|c|}{$-14.49 \mathrm{erg} \mathrm{cm}^{-2} \mathrm{~s}^{-1}$} & $\log \mathrm{F}(\mathrm{H} \beta)$ & \multicolumn{2}{|c|}{$-13.66 \mathrm{erg} \mathrm{cm}^{-2} \mathrm{~s}^{-1}$} \\
\hline
\end{tabular}



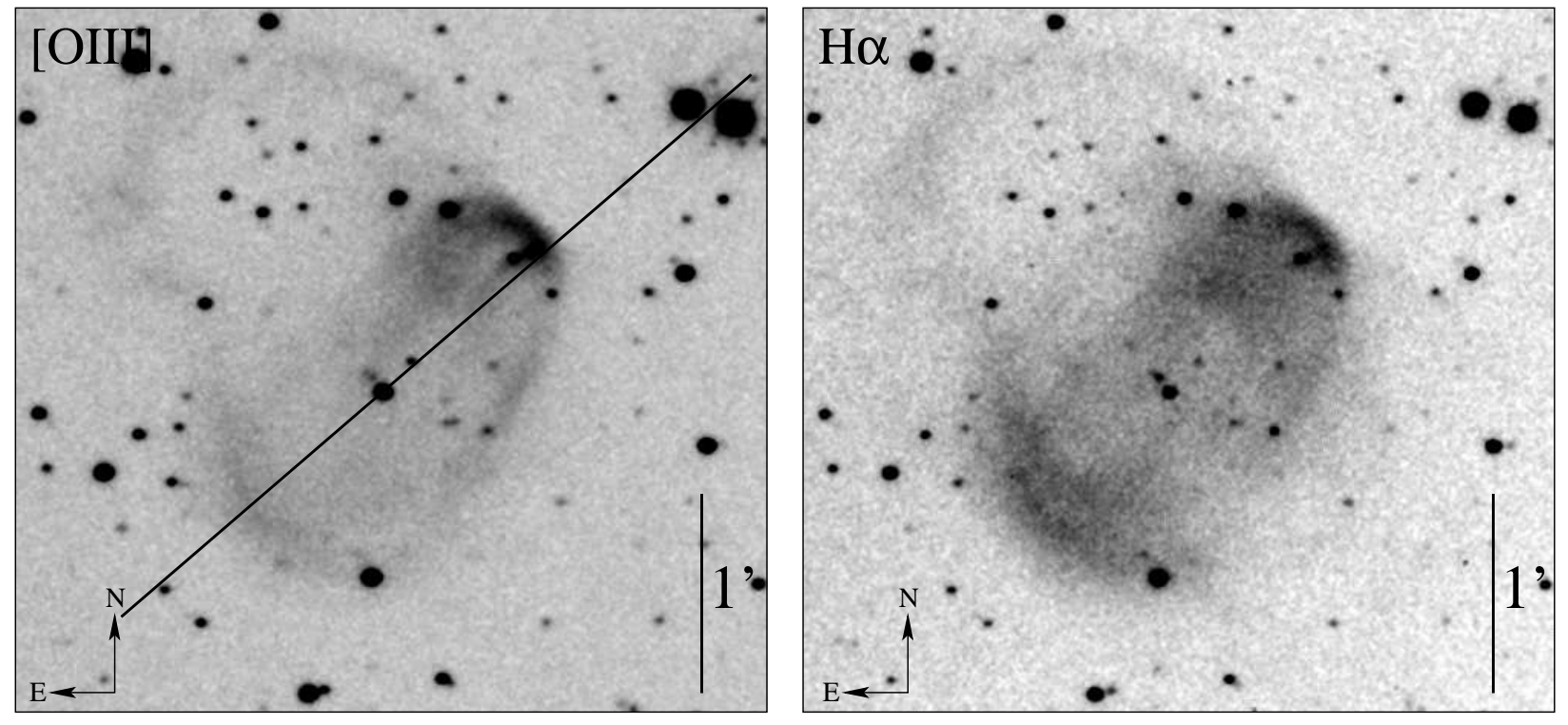

Fig. 2.- Images of $\mathrm{Pa} 5$ obtained at the OAN-SPM $84 \mathrm{~cm}$ telescope in the light of [O III $] 5007 \AA$ (left panel) and H $\alpha$ (right panel). Location of the long-slit is indicated on the $[\mathrm{O}$ III] image 

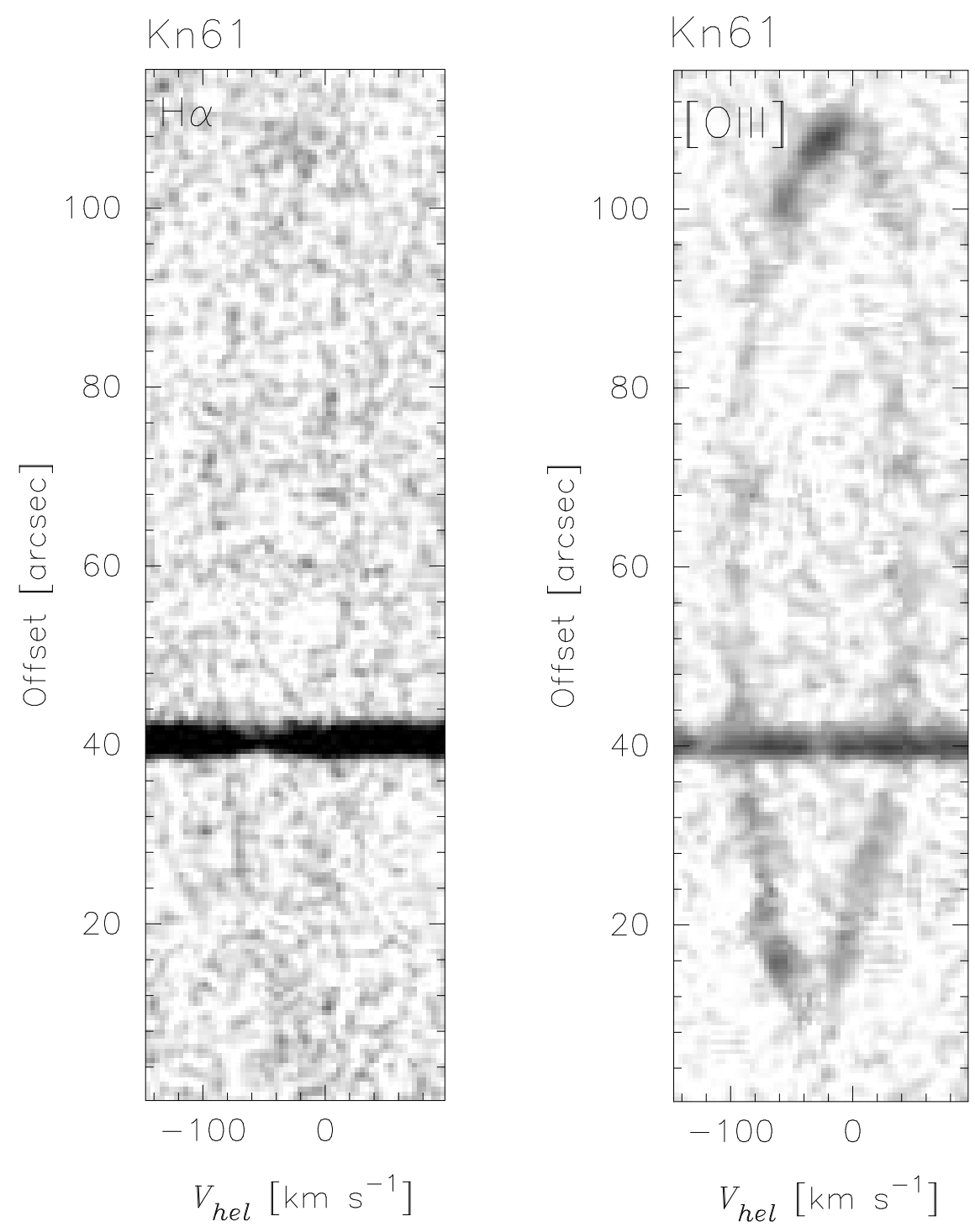

Fig. 3.- Kn 61 Bi-dimensional $\mathrm{P}-\mathrm{V}$ array, the continuum from a field star has not been subtracted 

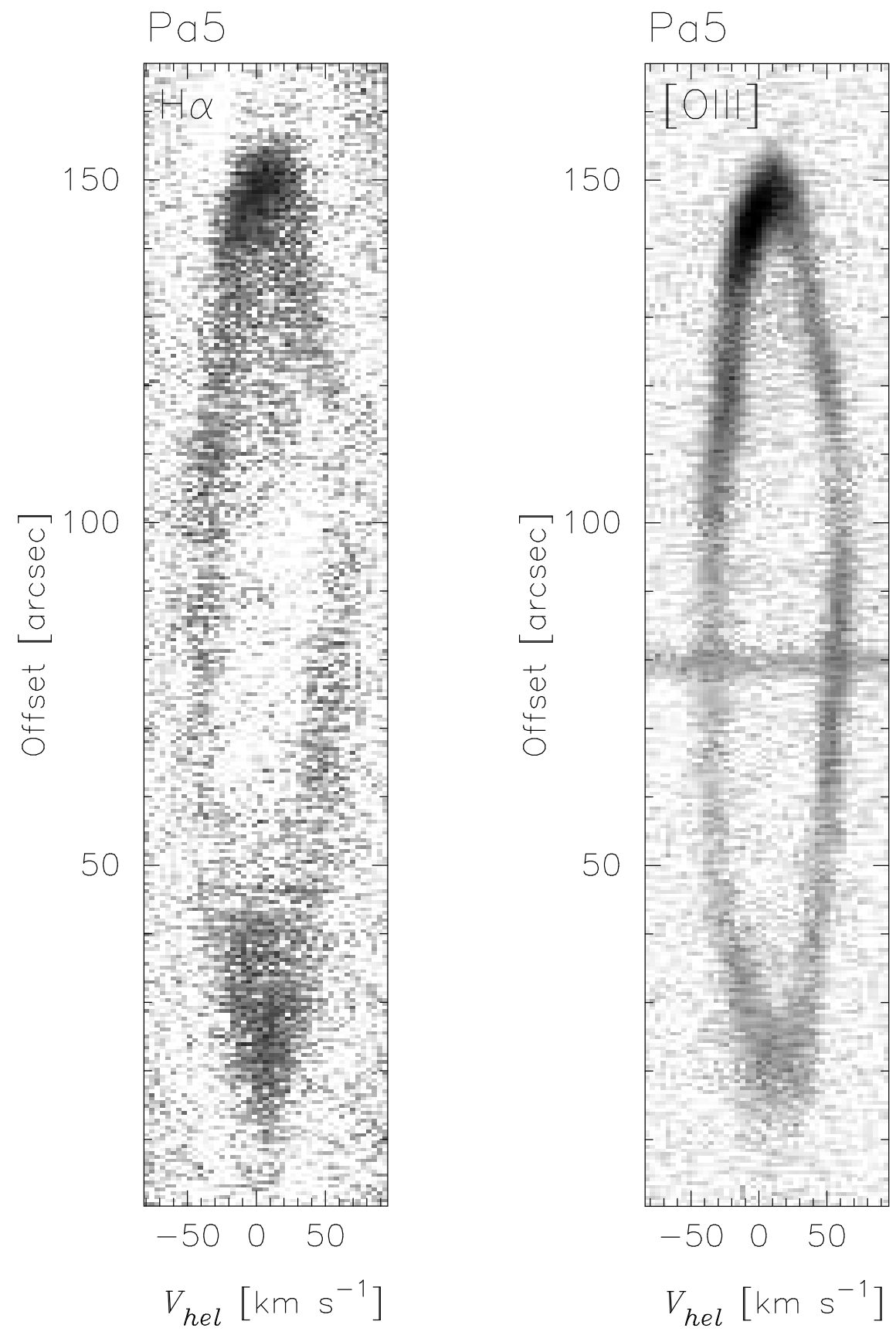

Fig. 4. - Pa 5 Bi-dimensional $\mathrm{P}-\mathrm{V}$ array. The continuum from the central star has been subtracted from the $\mathrm{H} \alpha \mathrm{P}-\mathrm{V}$ array. 

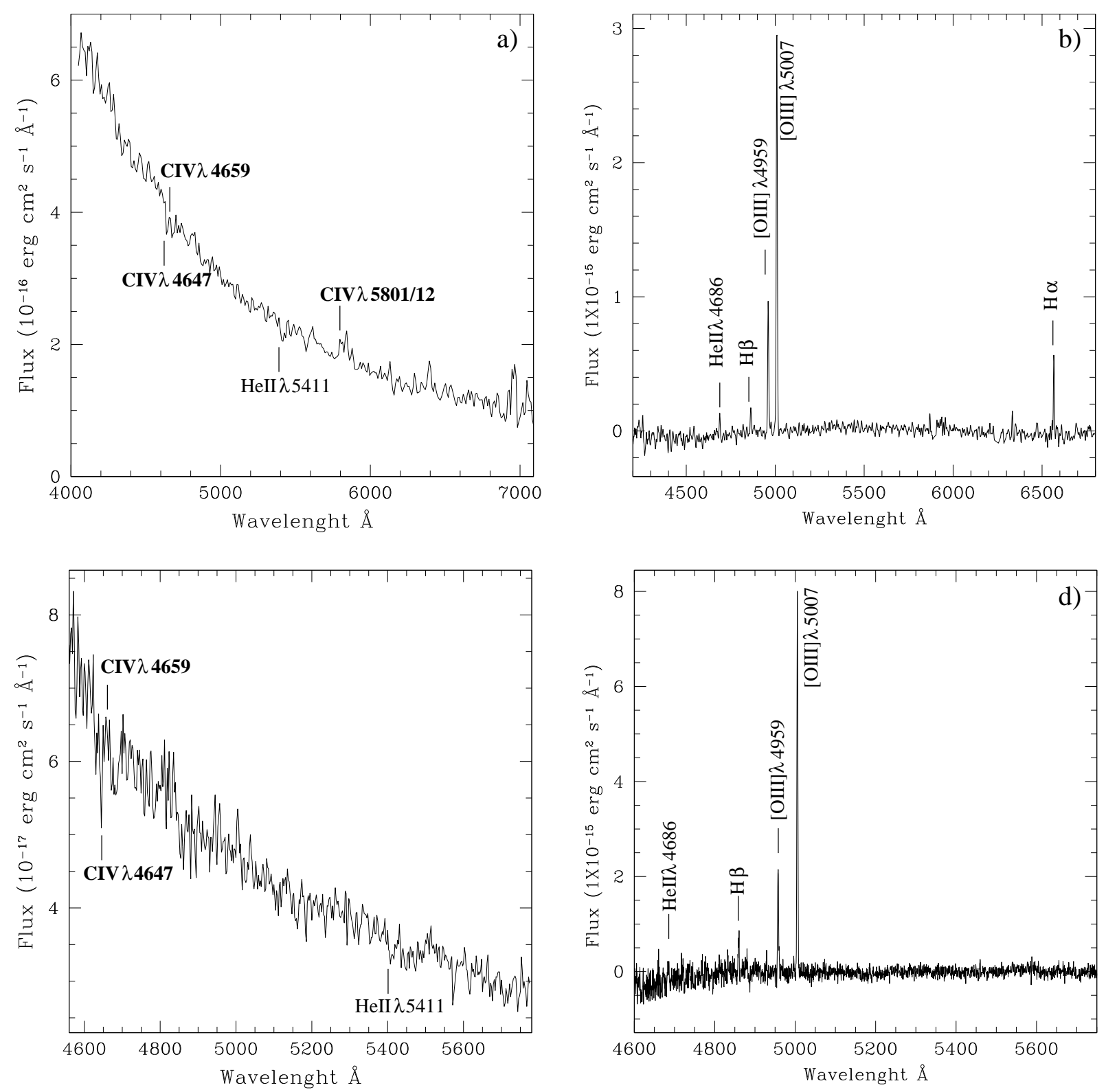

Fig. 5.- Panels a) and b): OAN-SPM one-dimensional stellar and nebular spectra of Kn 61, respectively, taken with a $600 \mathrm{l} \mathrm{mm}^{-1}$ grating. Panels c) and d): OAN-SPM one-dimensional stellar and nebular spectra of Kn 61, respectively, taken with the $1200 \mathrm{l} \mathrm{mm}^{-1}$ grating. 

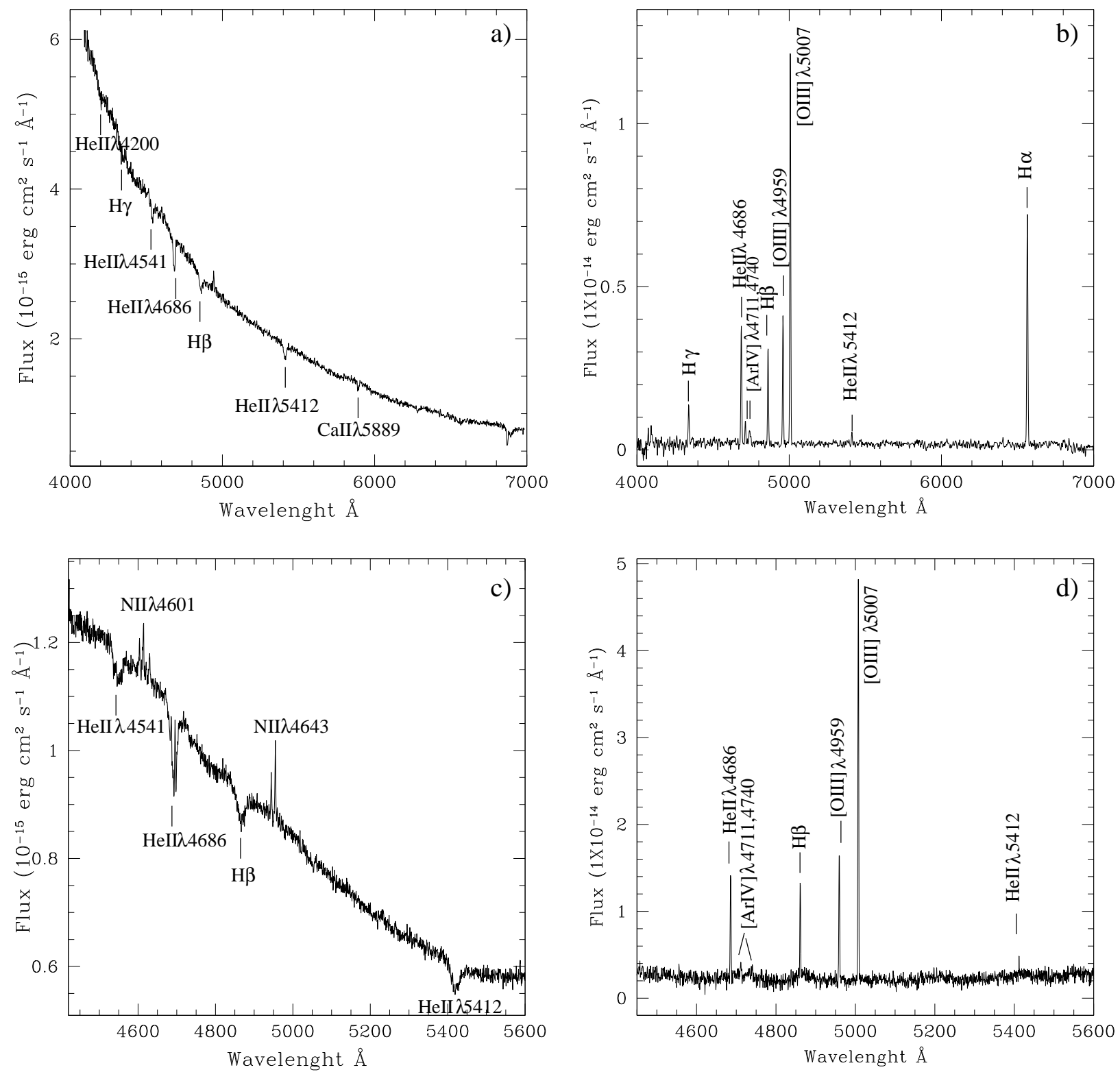

Fig. 6.- Panels a) and b): OAN-SPM one-dimensional stellar and nebular spectra of $\mathrm{Pa}$ 5, respectively, taken with a $600 \mathrm{l} \mathrm{mm}^{-1}$ grating. Panels c) and d): OAN-SPM one-dimensional stellar and nebular spectra of Pa 5, respectively, taken with the $1200 \mathrm{l} \mathrm{mm}^{-1}$ grating. 


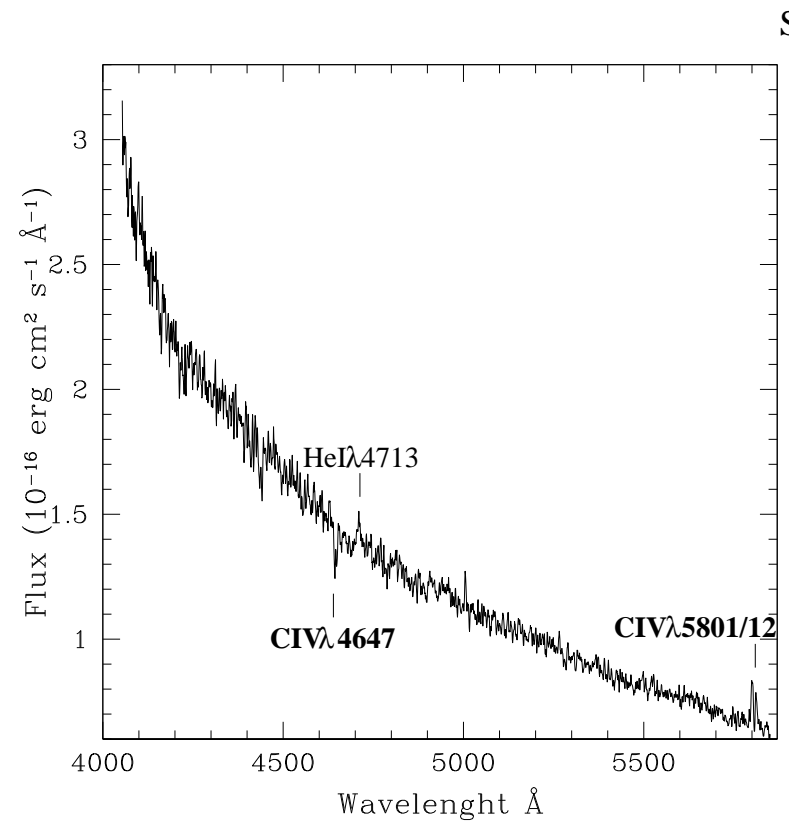

SAO-RAS

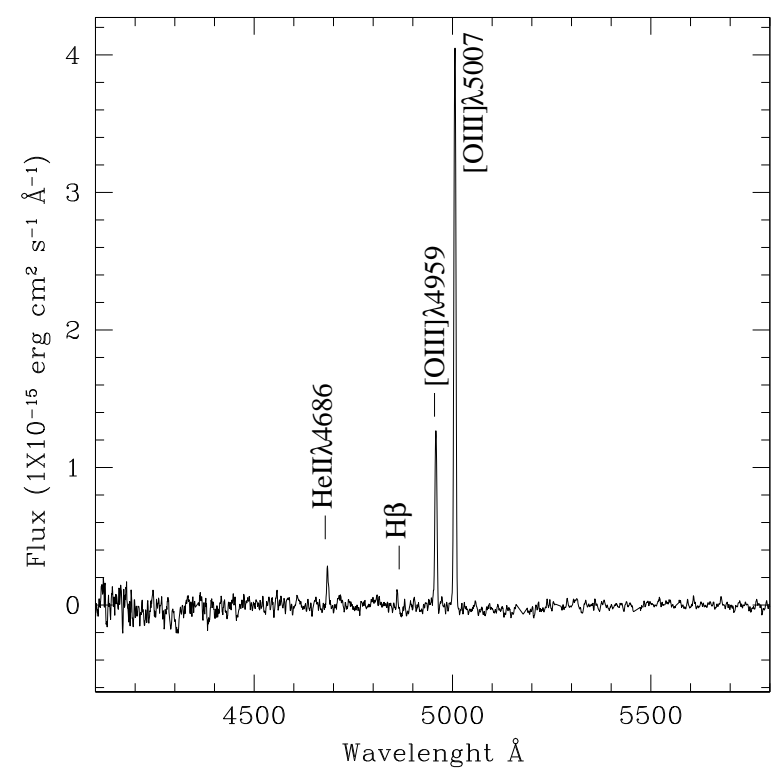

Fig. 7.- SAO-RAS spectra. Left panel) Stellar spectra of SDSS J192138.93+381857.2. Right Panel) Nebular spectrum of Kn 61 

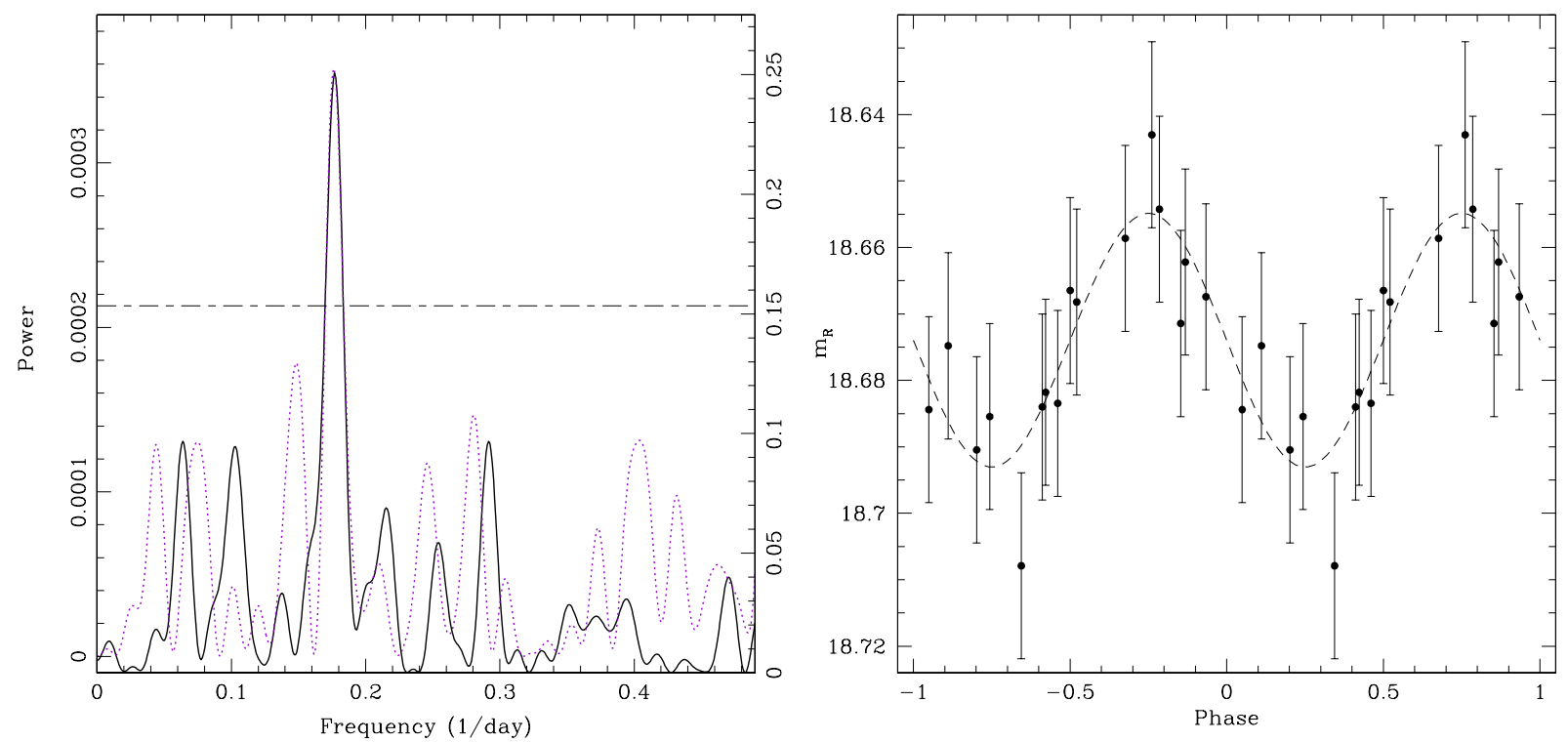

Fig. 8.- left panel: Power spectrum of the photometric light curve. The dotted line represents the raw power spectrum calculated by using average nightly brightness and its power scale is indicated by the left vertical axis. The solid line is the power spectrum obtained by CLEANing alias signals using the spectral window. Both curves are scaled to match the peak power, but the signal in the CLEANed spectrum is different, and its scale is indicated on the right-side axis. The power peaks at the $0.176 d^{-1}$ frequency. The horizontal dashed line indicates $99 \%$ confidence level of the raw power spectrum. right panel: The light curve of the Kn 61 central star folded with the 5.7 day period. The error bars correspond to the RMS of individual night light curves, the points correspond to the nightly brightness average. The best-fit sin curve is overlaid on the data. 

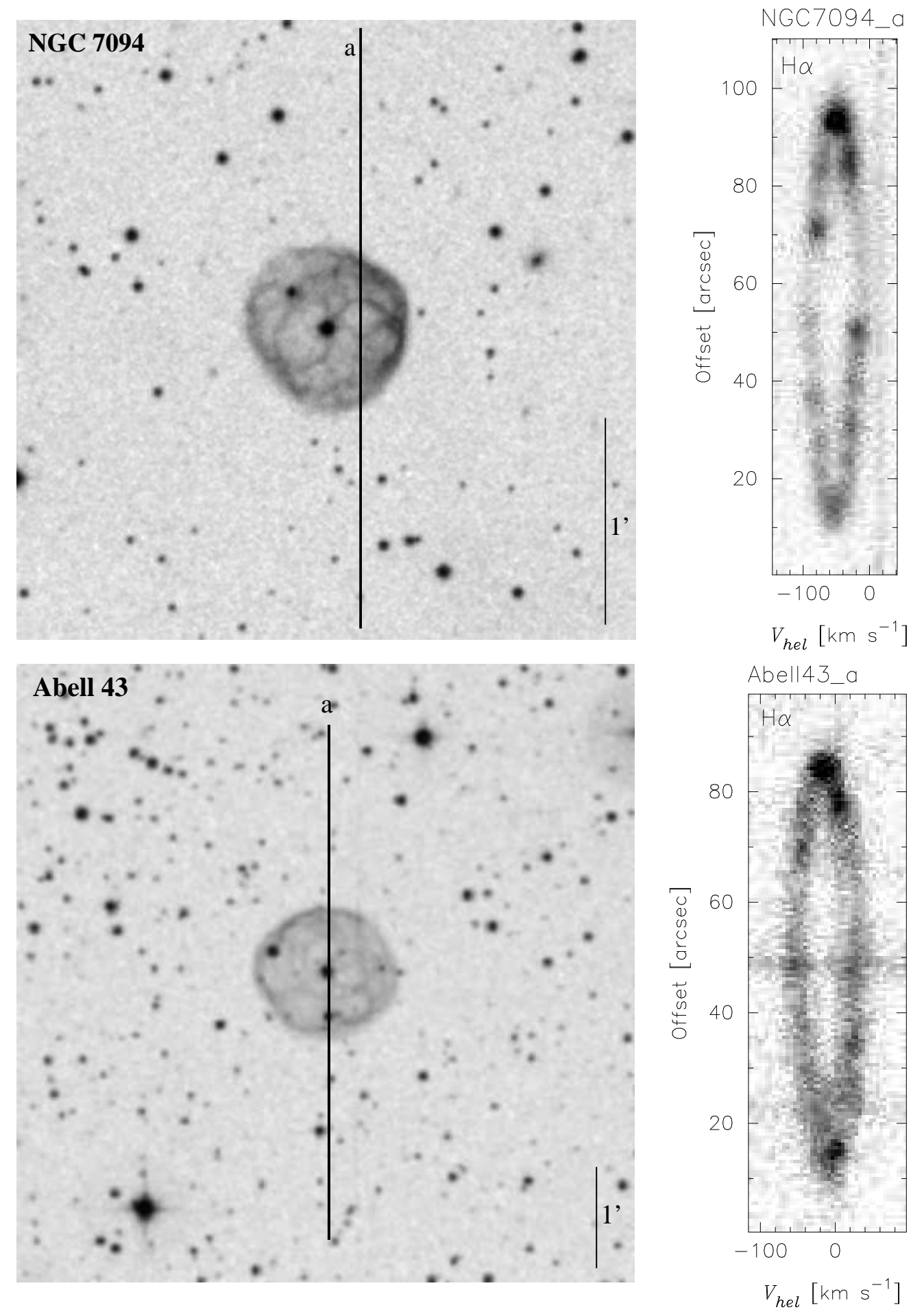

Fig. 9.- Images and long-slit spectra of NGC 7094 and Abell 43 taken from the SPM Kinematic Catalogue of Galactic Planetary Nebulae, http://kincatpn.astrosen.unam.mx/. Notice their similarity in both, images and spectra, with Kn 61 